\title{
HARMONIC SPINORS AND LOCAL DEFORMATIONS OF THE METRIC
}

\author{
Bernd Ammann, Mattias Dahl and Emmanuel Humbert
}

\begin{abstract}
Let $(M, g)$ be a compact Riemannian spin manifold. The Atiyah-Singer index theorem yields a lower bound for the dimension of the kernel of the Dirac operator. We prove that this bound can be attained by changing the Riemannian metric $g$ on an arbitrarily small open set.
\end{abstract}

\section{Introduction and statement of results}

Let $M$ be a spin manifold, we assume that all spin manifolds come equipped with a choice of orientation and spin structure. The Dirac operator $D^{g}$ of $(M, g)$ is a first order differential operator acting on sections of the spinor bundle associated to the spin structure on $M$. This is an elliptic, formally self-adjoint operator. If $M$ is compact, then the spectrum of $D^{g}$ is real, discrete, and the eigenvalues tend to plus and minus infinity. In this case, the operator $D^{g}$ is invertible if and only if 0 is not an eigenvalue, which is the same as vanishing of the kernel.

The Atiyah-Singer index theorem states that the index of the Dirac operator is equal to a topological invariant of the manifold,

$$
\operatorname{ind}\left(D^{g}\right)=\alpha(M),
$$

see, for example, [11, Theorem 16.6, p. 276]. Depending on the dimension $n$ of $M$ this formula has slightly different interpretations. To explain this interpretation, it is important to remark that we will always consider the spinor bundle as a complex vector bundle, similar results with different dimensions would also hold for the real spinor bundle or the $\mathrm{C} \ell_{n}$-linear spinor bundle. If $n$ is even there is a \pm -grading of the spinor bundle and the Dirac operator $D^{g}$ has a part $\left(D^{g}\right)^{+}$, which maps from positive to negative spinors. If $n \equiv 0,4 \bmod 8$ the index is integer-valued and computed as the dimension of the kernel minus the dimension of the cokernel of $\left(D^{g}\right)^{+}$. If $n \equiv 1,2 \bmod 8$ the index is $\mathbb{Z} / 2 \mathbb{Z}$-valued and given by the dimension modulo 2 of the kernel of $D^{g}$ (if $\left.n \equiv 1 \bmod 8\right)$ resp. $\left(D^{g}\right)^{+}($if $n \equiv 2 \bmod 8)$. In other dimensions the index is zero. In all dimensions, $\alpha(M)$ is a topological invariant depending only on the spin bordism class of $M$. In particular, $\alpha(M)$ does not depend on the metric, but it depends on the spin structure in dimension $n \equiv 1,2 \bmod 8$. For further details see [11, Chapter II, §7].

Received by the editors June 7, 2011.

2000 Mathematics Subject Classification. 53C27 (Primary) 55N22, 57R65 (Secondary).

Key words and phrases. Dirac operator, eigenvalue, surgery, index theorem. 
The index theorem implies a lower bound on the dimension of the kernel of $D^{g}$ which we can write succinctly as

$$
\operatorname{dim} \operatorname{ker} D^{g} \geq a(M),
$$

where

$$
a(M):=\left\{\begin{array}{ll}
|\widehat{A}(M)| & \text { if } n \equiv 0 \bmod 4 \\
1 & \text { if } n \equiv 1 \bmod 8 \text { and } \alpha(M) \neq 0 \\
2 & \text { if } n \equiv 2 \bmod 8 \text { and } \alpha(M) \neq 0 \\
0 & \text { otherwise. }
\end{array}\right. \text {. }
$$

If $M$ is not connected, then this lower bound can be improved by studying each connected component of $M$. For this reason we restrict to connected manifolds from now on.

Metrics $g$ for which equality holds in (1) are called $D$-minimal, see [3, Section 3]. The existence of $D$-minimal metrics on all connected compact spin manifolds was established in [1] following previous work in $[12,3]$. In this note we will strengthen this existence result by showing that one can find a $D$-minimal metric coinciding with a given metric outside a small open set. For a Riemannian manifold $(M, g)$ we denote by $U_{p}(r)$ the set of points for which the distance to the point $p$ is strictly less than $r$. We will prove the following theorem.

Theorem 1.1. Let $(M, g)$ be a compact connected Riemannian spin manifold of dimension $n \geq 2$. Let $p \in M$ and $r>0$. Then there is a D-minimal metric $\widetilde{g}$ on $M$ with $\widetilde{g}=g$ on $M \backslash U_{p}(r)$.

The new ingredient in the proof of this theorem is the use of the "invertible double" construction which gives a $D$-minimal metric on any spin manifold of the type $(-M) \# M$ where \# denotes connected sum and where $-M$ denotes $M$ equipped with the opposite orientation. For dimension $n \geq 5$, we can then use the surgery method from [3] with surgeries of codimension $\geq 3$. For $n=3$, 4 , we need the stronger surgery result of [1] preserving $D$-minimality under surgeries of codimension $\geq 2$. The case $n=2$ follows from [1] and classical facts about Riemann surfaces.

If a manifold has one $D$-minimal metric, then generic metrics are $D$-minimal, to formulate this precisely we introduce some notation. We denote by $\mathcal{R}\left(M, U_{p}(r), g\right)$ the set of all smooth Riemannian metrics on $M$ which coincide with the metric $g$ outside $U_{p}(r)$ and by $\mathcal{R}_{\min }\left(M, U_{p}(r), g\right)$ the subset of $D$-minimal metrics. From Theorem 1.1 it follows that a generic metric from $\mathcal{R}\left(M, U_{p}(r), g\right)$ is actually in $\mathcal{R}_{\min }\left(M, U_{p}(r), g\right)$, as made precise in the following corollary.

Corollary 1.1. Let $(M, g)$ be a compact connected Riemannian spin manifold of dimension $\geq 3$. Let $p \in M$ and $r>0$. Then $\mathcal{R}_{\min }\left(M, U_{p}(r), g\right)$ is open in the $C^{1}$-topology on $\mathcal{R}\left(M, U_{p}(r), g\right)$ and it is dense in all $C^{k}$-topologies, $k \geq 1$.

The proof follows ideas described in [2, Theorem 1.2] or [12, Proposition 3.1]. The first observation of the argument is that the eigenvalues of $D^{g}$ are continuous functions of $g$ in the $C^{1}$-topology, from which the property of being open follows. The second observation is that spectral data of $D^{g_{t}}$ for a linear family of metrics $g_{t}=(1-t) g_{0}+t g_{1}$ depends real analytically on the parameter $t$. If $g_{0} \in \mathcal{R}_{\min }\left(M, U_{p}(r), g\right)$ it follows that metrics $g_{t}$ with $t$ arbitrarily close to 1 are also in this set, from which we conclude the property of being dense. 


\section{Preliminaries}

2.1. Spin manifolds and spin structure preserving maps. An orientation on an $n$-dimensional manifold $M$ can be viewed as a refinement of the frame bundle $\mathrm{GL}(M)$ for the tangent bundle $T M$ to a sub-bundle $\mathrm{GL}_{+}(M)$ with structure group $\mathrm{GL}_{+}(n, \mathbb{R})$. Such a refinement exists if and only if the first Stiefel-Whitney class $w_{1}(T M)$ vanishes. Here the group $\mathrm{GL}_{+}(n, \mathbb{R})$ consists of all invertible $n \times n$-matrices with positive determinant and has fundamental group $\mathbb{Z}$ if $n=2$ and $\mathbb{Z} / 2 \mathbb{Z}$ if $n \geq 3$. Let $\widetilde{\mathrm{GL}_{+}}(n, \mathbb{R})$ be the unique connected double cover of $\mathrm{GL}_{+}(n, \mathbb{R})$.

A (topological) spin structure on an oriented manifold $M$ is a $\left(\widetilde{\mathrm{GL}_{+}}(n, \mathbb{R}) \rightarrow\right.$ $\operatorname{GL}(n, \mathbb{R}))$-equivariant lift of $\mathrm{GL}_{+}(M)$ to a bundle with the structure group $\widetilde{\mathrm{GL}_{+}}(n, \mathbb{R})$. Such a lift exists if and only if the second Stiefel-Whitney class $\left.W_{2}(T M)\right)$ vanishes.

If these structures exist they are in general not unique, the orientation can be chosen independently on each connected component of $M$, or equivalently the space of orientations on $M$ is an affine space for the $\mathbb{Z} / 2 \mathbb{Z}$-vector space $H^{0}(M, \mathbb{Z} / 2 \mathbb{Z})$. Similarly, the space of spin structures is an affine space for the $\mathbb{Z} / 2 \mathbb{Z}$-vector space $H^{1}(M, \mathbb{Z} / 2 \mathbb{Z})$.

As already mentioned we use the term "spin manifold" for a manifold together with the choice of an orientation and a spin structure.

If $f: M_{1} \rightarrow M_{2}$ is a diffeomorphism between two manifolds, any orientation and spin structure on $M_{2}$ pulls back to an orientation and spin structure on $M_{1}$. A diffeomorphism $f$ between two spin manifolds $M_{1}$ and $M_{2}$ is called a spin structure preserving diffeomorphism if the orientation and spin structure on $M_{1}$ coincide with the pullbacks from $M_{2}$.

If the manifold $M$ is further equipped with a Riemannian metric the above topological spin structure reduces to a geometrical spin structure which is a $(\operatorname{Spin}(n) \rightarrow$ $\mathrm{SO}(n))$-equivariant lift $\operatorname{Spin}(M)$ of the bundle $\mathrm{SO}(M)$ of oriented orthonormal frames of the tangent bundle. The spinor bundle $\Sigma M$ on $M$ is a vector bundle associated to $\operatorname{Spin}(M)$, it has a natural first order elliptic operator $D: \Gamma(\Sigma M) \rightarrow \Gamma(\Sigma M)$, see, for example, [8] for details. Any spin structure preserving diffeomorphism $f: M_{1} \rightarrow$ $M_{2}$ which is also an isometry induces an isomorphism between the spinor bundles $f_{*}: \Sigma M_{1} \rightarrow \Sigma M_{2}$ which is compatible with the Dirac operators in the sense that all sections $\varphi$ of $\Sigma M_{1}$ satisfy $D^{M_{2}}\left(f_{*} \circ \varphi \circ f^{-1}\right)=f_{*} \circ\left(D^{M_{1}} \varphi\right) \circ f^{-1}$.

If $W$ is a manifold with boundary $\partial W=M$, then an orientation and spin structure on $W$ induce an orientation and a spin structure on $M$. Conversely, if an orientation and a spin structure on $M$ are given, then there is a unique orientation and spin structure on $W=M \times[0,1]$ such that the restricted structures on $M \cong M \times\{1\}$ coincide with the given ones. The boundary component $M \times\{0\}$ is obviously diffeomorphic to $M$ as well, but the restriction of the orientation of $M \times[0,1]$ is the opposite of the orientation of $M$. We write $-M:=M \times\{0\}$ for the spin manifold with this opposite orientation and the spin structure obtained from $M \times[0,1]$.

2.2. The invertible double. Let $N$ be a compact connected spin manifold with boundary. The double of $N$ is formed by gluing $N$ and $-N$ along the common boundary $\partial N$ and is denoted by $(-N) \cup_{\partial N} N$. If $N$ is equipped with a Riemannian metric which has product structure near the boundary, then this metric naturally gives a metric on $(-N) \cup_{\partial N} N$. The spin structures can be glued together to obtain a spin structure on $(-N) \cup_{\partial N} N$. The spinor bundle of $(-N) \cup_{\partial N} N$ is obtained by 
gluing the spinor bundle of $N$ with the spinor bundle of $-N$ along their common boundary $\partial N$. It is straightforward to check that the appropriate gluing map is the map used in [6, Chapter 9].

The Dirac operator on $(-N) \cup_{\partial N} N$ is invertible due to the following argument. Assume that a spinor field $\varphi$ is in the kernel of the Dirac operator on $(-N) \cup_{\partial N} N$. The restriction $\left.\varphi\right|_{-N}$ can be "reflected along $\partial N$ " to a spinor field $\tilde{\varphi}$ on $N$ as indicated in the appendix. On the boundary $\partial N$ one has $\left.\tilde{\varphi}\right|_{N}=\left.\nu \cdot \varphi\right|_{N}$ and thus $\left.\nu \cdot \tilde{\varphi}\right|_{N}=-\left.\varphi\right|_{N}$ for the exterior unit normal field $\nu$ on $\partial N$, see Lemma A.2. Green's formula for the Dirac operator yields

$$
0=\int_{N}\langle D \tilde{\varphi}, \varphi\rangle-\int_{N}\langle\tilde{\varphi}, D \varphi\rangle=\int_{\partial N}\langle\nu \cdot \tilde{\varphi}, \varphi\rangle=-\left\|\left.\varphi\right|_{\partial N}\right\|_{L^{2}(\partial N)}^{2} .
$$

Thus $\left.\varphi\right|_{\partial N}=0$, and by the weak unique continuation property of the Dirac operator it follows that $\varphi=0$. For more details on this argument see [6, Chapter 9] and [5, Proposition 1.4]. In the appendix we also show that the doubling construction of [6, Chapter 9] coincides with the spinor bundle and Dirac operator on the doubled manifold.

Proposition 2.1. Let $(M, g)$ be a compact connected Riemannian spin manifold. Let $p \in M$ and $r>0$. Let $(-M) \# M$ be the connected sum formed at the points $p \in M$ and $p \in-M$. Then there is a metric on $(-M) \# M$ with invertible Dirac operator which coincides with $g$ outside $U_{p}(r)$

This proposition is proved by applying the double construction to the manifold with boundary $N=M \backslash U_{p}(r / 2)$, where $N$ is equipped with a metric we get by deforming the metric $g$ on $U_{p}(r) \backslash U_{p}(r / 2)$ to become a product near the boundary.

Metrics with invertible Dirac operator are obviously $D$-minimal, so the metric provided by Proposition 2.1 is $D$-minimal.

\section{Proof of Theorem 1.1}

Let $M$ and $N$ be compact spin manifolds of dimension $n$. Recall that a spin bordism from $M$ to $N$ is a manifold with boundary $W$ of dimension $n+1$ together with a spin structure preserving diffeomorphism from $N \amalg(-M)$ to the boundary of $W$. The manifolds $M$ and $N$ are said to be spin bordant if such a bordism exists.

For the proof of Theorem 1.1 we have to distinguish several cases.

\subsection{Proof of Theorem 1.1 in dimension $n \geq 5$.}

Proof. To prove the Gromov-Lawson conjecture, Stolz [13] showed that any compact spin manifold with vanishing index is spin bordant to a manifold of positive scalar curvature. Using this, we see that $M$ is spin bordant to a manifold $N$ which has a $D$-minimal metric $h$, where the manifold $N$ is not necessarily connected. For details see [3, Proposition 3.9].

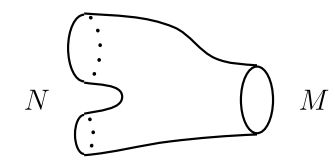


By removing an open ball from the interior of a spin bordism from $M$ to $N$ we get that $N \amalg(-M)$ is spin bordant to the sphere $S^{n}$.

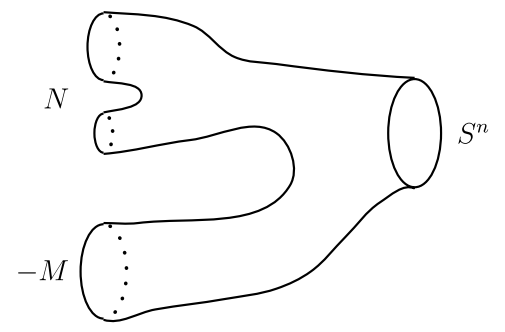

Since $S^{n}$ is simply connected and $n \geq 5$ it follows from [11, Proof of Theorem 4.4, p. 300] that $S^{n}$ can be obtained from $N \amalg(-M)$ by a sequence of surgeries of codimension at least 3. By making $r$ smaller and possibly move the surgery spheres slightly we may assume that no surgery hits $U_{p}(r) \subset M$. We obtain a sequence of manifolds $N_{0}, N_{1}, \ldots, N_{k}$, where $N_{0}=N \amalg(-M), N_{k}=S^{n}$, and $N_{i+1}$ is obtained from $N_{i}$ by a surgery of codimension at least 3 .

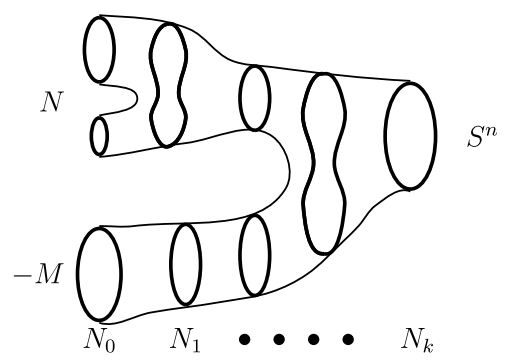

Since the surgeries do not hit $U_{p}(r) \subset M \subset N \amalg(-M)=N_{0}$ we can consider $U_{p}(r)$ as a subset of every $N_{i}$. We define the sequence of manifolds $N_{0}^{\prime}, N_{1}^{\prime}, \ldots, N_{k}^{\prime}$ by forming the connected sum $N_{i}^{\prime}=M \# N_{i}$ at the points $p$. Then $N_{0}^{\prime}=N \amalg(-M) \# M$, $N_{k}^{\prime}=S^{n} \# M=M$, and $N_{i+1}^{\prime}$ is obtained from $N_{i}^{\prime}$ by a surgery of codimension at least 3 which does not hit $M \backslash U_{p}(r)$.

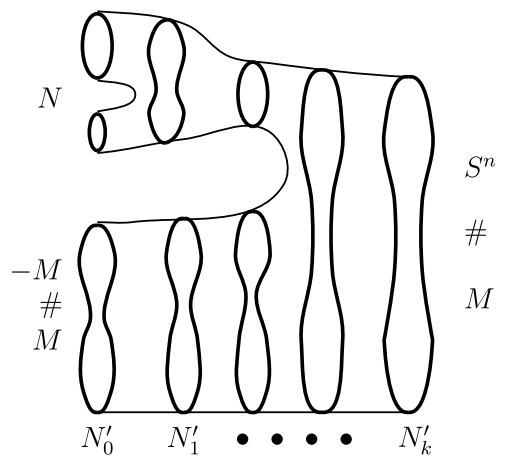

We now equip $N_{0}^{\prime}$ with a Riemannian metric. On $N$ we choose a $D$-minimal metric. The manifold $(-M) \# M$ has vanishing index, so a $D$-minimal metric is a metric with an invertible Dirac operator. From Proposition 2.1, we know that there exists such a metric on $(-M) \# M$ which coincides with $g$ outside $U_{p}(r)$. Note that here we use the assumption that $M$ is connected. Together we get a $D$-minimal metric $g_{0}^{\prime}$ on $N_{0}^{\prime}$. 
From [3, Proposition 3.6] we know that the property of being $D$-minimal is preserved under surgery of codimension at least 3 . We apply the surgery procedure to $g_{0}^{\prime}$ to produce a sequence of $D$-minimal metrics $g_{i}^{\prime}$ on $N_{i}^{\prime}$. Since the surgery procedure of [3, Theorem 1.2] does not affect the Riemannian metrics outside arbitrarily small neighborhoods of the surgery spheres we may assume that all $g_{i}^{\prime}$ coincide with $g$ on $M \backslash U_{p}(r)$. The Theorem is proved by choosing $\tilde{g}=g_{k}^{\prime}$ on $N_{k}^{\prime}=M$.

\subsection{Proof of Theorem 1.1 in dimensions $n=3$ and $n=4$.}

Proof. In these cases the argument works almost the same, except that we can only conclude that $S^{n}$ is obtained from $N \amalg(-M)$ by surgeries of codimension at least 2 , see [9, VII, Theorem 3] for $n=3$ and [10, VIII, Proposition 3.1] for $n=4$. To take care of surgeries of codimension 2 we use [1, Theorem 1.2]. Since this surgery construction affects the Riemannian metric only in a small neighborhood of the surgery sphere we can finish the proof as described in the case $n \geq 5$.

Alternatively, it is straightforward to adapt the perturbation proof by Maier [12] to prove Theorem 1.1 in dimensions 3 and 4 .

\subsection{Proof of Theorem 1.1 in dimension $n=2$.}

Proof. The argument in the case $n=2$ is different. Assume that a metric $g$ on a compact surface with chosen spin structure is given. In [1, Theorem 1.1] it is shown that for any $\varepsilon>0$ there is a $D$-minimal metric $\hat{g}$ with $\|g-\hat{g}\|_{C^{1}}<\varepsilon$. Using the following Lemma 3.1, we see that for $\varepsilon>0$ sufficiently small, there is a spin structure preserving diffeomorphism $\psi: M \rightarrow M$ such that $\tilde{g}:=\psi^{*} \hat{g}$ is conformal to $g$ on $M \backslash U_{p}(r)$. As the dimension of the kernel of the Dirac operator is preserved under spin structure preserving conformal diffeomorphisms, $\tilde{g}$ is $D$-minimal as well.

Lemma 3.1. Let $M$ be a compact surface with a Riemannian metric $g$ and a spin structure. Then for any $r>0$ there is an $\varepsilon>0$ with the following property: For any $\hat{g}$ with $\|g-\hat{g}\|_{C^{1}}<\varepsilon$ there is a spin structure preserving diffeomorphism $\psi: M \rightarrow M$ such that $\tilde{g}:=\psi^{*} \hat{g}$ is conformal to $g$ on $M \backslash U_{p}(r)$.

To prove the lemma one has to show that a certain differential is surjective. This proof can be carried out in different mathematical languages. One alternative is to use Teichmüller theory formulated in terms of quadratic differentials, we will use a presentation in terms of Riemannian metrics following [14].

Sketch of Proof of Lemma 3.1. If $g_{1}$ and $g_{2}$ are metrics on $M$, then we say that $g_{1}$ is Teichmüller equivalent to $g_{2}$ if there is a diffeomorphism $\psi: M \rightarrow M$ such that $\psi$ is homotopic to the identity and $\psi^{*} g_{2}$ is conformal to $g_{1}$. This is an equivalence relation on the set of metrics on $M$, and the equivalence class of $g_{1}$ is denoted by $\Phi\left(g_{1}\right)$. Let $\mathcal{T}$ be the set of equivalence classes, this is the Teichmüller space which has a natural structure of a smooth finite-dimensional manifold. Note that any diffeomorphism $\psi: M \rightarrow M$ homotopic to the identity is also isotopic to the identity, i.e. the homotopy can be chosen as a path in the diffeomorphism group, see, e.g., [7]. As along this path, the spin structure is preserved, $\psi$ perserves the spin structure.

Showing the lemma is thus equivalent to showing that $\Phi\left(\mathcal{R}\left(M, U_{p}(r), g\right)\right)$ is a neighborhood of $\Phi(g)$ in $\mathcal{T}$. 
Variations of metrics are given by symmetric $(2,0)$-tensors, that is, by sections of $S^{2} T^{*} M$. The tangent space of $\mathcal{T}$ can be identified with the space of transverse $(=$ divergence free) traceless sections,

$$
S^{T T}:=\left\{h \in \Gamma\left(S^{2} T^{*} M\right) \mid \operatorname{div}^{g} h=0, \operatorname{tr}^{g} h=0\right\},
$$

see, for example, [4, Lemma 4.57] and [14].

The two-dimensional manifold $M$ has a complex structure which is denoted by $J$. The map $H: T^{*} M \rightarrow S^{2} T^{*} M$ defined by $H(\alpha):=\alpha \otimes \alpha-\alpha \circ J \otimes \alpha \circ J$ is quadratic, it is 2-to- 1 outside the zero section, and its image are the trace free symmetric tensors. Furthermore $H(\alpha \circ J)=-H(\alpha)$. Hence by polarization we obtain an isomorphism of real vector bundles from $T^{*} M \otimes_{\mathbb{C}} T^{*} M$ to the trace free part of $S^{2} T^{*} M$. Here the complex tensor product is used when $T^{*} M$ is considered as a complex line bundle using $J$. A trace-free section of $S^{2} T^{*} M$ is divergence free if and only if the corresponding section $T^{*} M \otimes_{\mathbb{C}} T^{*} M$ is holomorphic, see [14, p. 45-46]. We get that $S^{T T}$ is finite dimensional, and it follows that $\mathcal{T}$ is finite dimensional.

In order to show that $\Phi\left(\mathcal{R}\left(M, U_{p}(r), g\right)\right)$ is a neighborhood of $\Phi(g)$ in $\mathcal{T}$ we show that the differential $d \Phi: T \mathcal{R}\left(M, U_{p}(r), g\right) \rightarrow T \mathcal{T}$ is surjective at $g$. Using the above identification $T \mathcal{T}=S^{T T}, d \Phi$ is just orthogonal projection from $\Gamma\left(S^{2} T^{*} M\right)$ to $S^{T T}$.

Assume that $h_{0} \in S^{T T}$ is orthogonal to $d \Phi\left(T \mathcal{R}\left(M, U_{p}(r), g\right)\right)$. Then $h_{0}$ is $L^{2}$-orthogonal to $T \mathcal{R}\left(M, U_{p}(r), g\right)$. As $\left.T \mathcal{R}\left(M, U_{p}(r), g\right)\right)$ consists of all sections of $S^{2} T^{*} M$ with support in $U_{p}(r)$ we conclude that $h_{0}$ vanishes on $U_{p}(r)$. Since $h_{0}$ can be identified with a holomorphic section of $T^{*} M \otimes_{\mathbb{C}} T^{*} M$ we see that $h_{0}$ vanishes everywhere on $M$. The surjectivity of $d \Phi$ and the lemma follow.

\section{Appendix A. Notes about reflections at hypersurfaces and the doubling construction}

Let $M$ be a connected Riemannian spin manifold, with a reflection $\varphi$ at a hyperplane $N$. That is $\varphi$ is an isometry with fixed point set $N$, orientation reversing, and $N$ separates $M$ into two components. Let $-M$ be the manifold $M$ with the opposite orientation, i.e., $\varphi: M \rightarrow-M$ is orientation preserving. It is also required that $\varphi$ preserves the spin structure. The reflection $\varphi$ lifts to the frame bundle by mapping the frame $\mathcal{E}=\left(e_{1}, \ldots, e_{n}\right)$ to $\varphi_{*} \mathcal{E}:=\left(-d \varphi\left(e_{1}\right), d \varphi\left(e_{2}\right), \ldots, d \varphi\left(e_{n}\right)\right)$, so $\varphi_{*}: \mathrm{SO}(M) \rightarrow \mathrm{SO}(M)$. This map $\varphi_{*}$ is not $\mathrm{SO}(n)$ equivariant, but if we define $J=\operatorname{diag}(-1,1,1,1, \ldots 1)$, then

$$
\varphi_{*}(\mathcal{E} A)=\varphi_{*}(\mathcal{E}) J A J .
$$

If $\mathcal{E}$ is a frame over $N$ whose first vector is normal to $N$, then $\varphi_{*}(\mathcal{E})=\mathcal{E}$.

The above-mentioned compatibility with the spin structure is the fact that the pullback of the double covering $\vartheta: \operatorname{Spin}(M) \rightarrow \operatorname{SO}(M)$ via $\varphi_{*}$ is again the covering $\operatorname{Spin}(M) \rightarrow \operatorname{SO}(M)$. In other words, a lift $\widetilde{\varphi}_{*}: \operatorname{Spin}(M) \rightarrow \operatorname{Spin}(M)$ can be chosen such that $\vartheta \circ \widetilde{\varphi}_{*}=\varphi_{*} \circ \vartheta$. This implies that $\left(\widetilde{\varphi}_{*}\right)^{2}= \pm \operatorname{Id}$. Choose $\widetilde{\mathcal{E}} \in \operatorname{Spin}(M)$ over $N$, such that the first vector of $\vartheta(\tilde{\mathcal{E}})$ is normal to $N$. Then $\widetilde{\varphi}_{*}(\widetilde{\mathcal{E}})= \pm \widetilde{\mathcal{E}}$, thus $\left(\widetilde{\varphi}_{*}\right)^{2}(\widetilde{\mathcal{E}})=\widetilde{\mathcal{E}}$. It follows that $\left(\widetilde{\varphi}_{*}\right)^{2}=\mathrm{Id}$.

The conjugation with $J$ is an automorphism of $\mathrm{SO}(n)$ and lifts to $\operatorname{Spin}(n) \subset \mathrm{Cl}_{n}$, as a conjugation with $E_{1}:=(1,0 \ldots, 0)$ in the Clifford algebra sense. We therefore 
have

$$
\widetilde{\varphi}_{*}(\widetilde{\mathcal{E}} B)=\widetilde{\varphi}_{*}(\widetilde{\mathcal{E}})\left(-E_{1} B E_{1}\right) .
$$

Let $\sigma: \mathrm{Cl}_{n} \rightarrow \operatorname{End}\left(\Sigma_{n}\right)$ be an irreducible representation of the Clifford algebra. We set $\Sigma M:=\operatorname{Spin}(M) \times_{\sigma} \Sigma_{n}$.

Lemma A.1 (Lift to the spinor bundle). The map

$$
\operatorname{Spin}(M) \times \Sigma_{n} \ni(\widetilde{\mathcal{E}}, \rho) \mapsto\left(\widetilde{\varphi}_{*} \widetilde{\mathcal{E}}, \sigma\left(E_{1}\right) \rho\right) \in \operatorname{Spin}(M) \times \Sigma_{n}
$$

is compatible with the equivalence relation given by $\sigma$. Thus it descends to a map

$$
\varphi_{\#}: \Sigma M=\operatorname{Spin}(M) \times_{\sigma} \Sigma_{n} \rightarrow \Sigma M=\operatorname{Spin}(M) \times_{\sigma} \Sigma_{n} .
$$

Proof. $\left(\widetilde{\mathcal{E}} B, \sigma^{-1}(B) \rho\right)$ is mapped to

$$
\left.\left(\widetilde{\varphi}_{*}(\widetilde{\mathcal{E}} B), \sigma\left(E_{1}\right) \sigma^{-1}(B) \rho\right)=\widetilde{\varphi}_{*}(\widetilde{\mathcal{E}})\left(-E_{1} B E_{1}\right), \sigma\left(\left(-E_{1} B E_{1}\right)^{-1}\right) \sigma\left(E_{1}\right) \rho\right) .
$$

Obviously $\left(\varphi_{\#}\right)^{2}=-\mathrm{Id}$, and $\varphi_{\#}: \Sigma_{p} M \rightarrow \Sigma_{\varphi(p)} M$. In even dimensions $\varphi_{\#}$ maps positive spinors to negative ones and vice versa.

Lemma A.2 (On the fixed point set $N)$. Assume that $\left.\psi \in \Sigma M\right|_{N}$. Then $\varphi_{\#}(\psi)=$ $\pm \nu \cdot \psi$ for a unit normal vector $\nu$ of $N$ in $M$. The sign depends on the choice of $\nu$ and the choice of the lift $\tilde{\varphi}_{*}$.

Proof. Choose $\widetilde{\mathcal{E}} \in \operatorname{Spin}(M)$ over the base point of $\psi$, such that $\nu$ is the first vector of $\vartheta(\tilde{\mathcal{E}})$. Determine $\rho \in \Sigma_{n}$ such that $(\widetilde{\mathcal{E}}, \rho)$ represents $\psi$. Then $\varphi_{\#}(\psi)$ is represented by $( \pm \widetilde{\mathcal{E}}, \nu \cdot \rho)$.

Lemma A.3 (Compatibility with the Clifford action).

$$
d \varphi(X) \cdot \varphi_{\#}(\psi)=-\varphi_{\#}(X \cdot \psi)
$$

for $X \in T_{p} M, \psi \in \Sigma_{p} M$.

Proof. We view $T_{p} M$ as an associated bundle to $\operatorname{Spin}(M)$. Then $d \varphi([\widetilde{\mathcal{E}}, v])=$ $\left[\widetilde{\varphi}_{*}(\mathcal{E}), J v\right]$. Thus

$$
\begin{aligned}
d \varphi([\widetilde{\mathcal{E}}, v]) \cdot \varphi_{\#}([\widetilde{\mathcal{E}}, \rho]) & =\left[\widetilde{\varphi}_{*}(\widetilde{\mathcal{E}}), \sigma(J v) \sigma\left(E_{1}\right) \rho\right] \\
& =\left[\widetilde{\varphi}_{*}(\widetilde{\mathcal{E}}),-\sigma\left(E_{1}\right) \sigma(v) \rho\right] \\
& =-\varphi_{\#}([\widetilde{\mathcal{E}}, v] \cdot[\widetilde{\mathcal{E}}, \rho]) .
\end{aligned}
$$

Here we used that $J v=E_{1} \cdot v \cdot E_{1}$ in $\mathrm{Cl}_{n}$.

Lemma A.4. Let $X \in T_{p} M, \psi \in \Gamma(\Sigma M)$. Then

$$
\nabla_{d \varphi(X)} \varphi_{\#}(\psi)=\varphi_{\#}\left(\nabla_{X} \psi\right) .
$$

Proof. The differential of $\varphi_{*}: \mathrm{SO}(M) \rightarrow \mathrm{SO}(M)$ maps $T \mathrm{SO}(M)$ to $T \mathrm{SO}(M)$. The connection 1-form $\omega: \mathrm{SO}(M) \rightarrow \mathfrak{s o}(n)$ then pulls back according to

$$
\omega\left(\left(d\left(\varphi_{*}\right)\right)(Y)\right)=J \omega(Y) J
$$

for $Y \in T_{\mathcal{E}} \mathrm{SO}(M)$, a lift of $X \in T_{M}$ under the projection $\mathrm{SO}(M) \rightarrow M$. We lift this to a connection 1-form $\tilde{\omega}: \operatorname{Spin}(M) \rightarrow \mathrm{Cl}_{n}$, which thus transforms as

$$
\tilde{\omega}\left(\left(d\left(\tilde{\varphi}_{*}\right)\right)(\tilde{Y})\right)=-E_{1} \omega(\tilde{Y}) E_{1}
$$


where $\tilde{Y} \in T \operatorname{Spin}(M)$ is a lift of $Y$. And this induces the relation

$$
\nabla_{d \varphi(X)} \varphi_{\#}(\psi)=\varphi_{\#}\left(\nabla_{X} \psi\right) \text {. }
$$

We obtain

$$
\begin{aligned}
\varphi_{\#}(D \psi) & =\sum_{i} \varphi_{\#}\left(e_{i} \cdot \nabla_{e_{i}} \psi\right) \\
& =-\sum_{i} d \varphi\left(e_{i}\right) \cdot \varphi_{\#}\left(\nabla_{e_{i}} \psi\right) \\
& =-\sum_{i} d \varphi\left(e_{i}\right) \cdot \nabla_{d \varphi\left(e_{i}\right)} \varphi_{\#} \psi \\
& =-D \varphi_{\#} \psi
\end{aligned}
$$

This formula can also be read as

$$
D \psi=\varphi_{\#} D \varphi_{\#} \psi
$$

As a conclusion we obtain the following proposition.

Proposition A.1. If one constructs the double for a manifold with the classical spinor bundle and Dirac operator as in [6, Theorem 9.3], then we obtain the classical spinor bundle and the classical Dirac operator on the double.

To prove the proposition one has to compare the definitions in [6] with ours. The map $\varphi_{\#}: \Sigma_{p}^{+} M \rightarrow \Sigma_{\varphi(p)}^{-} M$ corresponds to the map $G$ in [6]. It follows that $G^{-1}$ corresponds to $-\varphi_{\#}: \Sigma_{p}^{-} M \rightarrow \Sigma_{\varphi(p)}^{+} M$. In [6], the map $G$ is used to identify $\Sigma_{p}^{+} M$ with $\Sigma_{\varphi(p)}^{-} M$. Pay attention that with respect to this identification, the map $\varphi_{\#}$ : $\Sigma_{p}^{+} M \rightarrow \Sigma_{\varphi(p)}^{-} M$ is the identity, whereas $\varphi_{\#}: \Sigma_{p}^{-} M \rightarrow \Sigma_{\varphi(p)}^{+} M$ is - Id. Equation (2) says that this identification is compatible with the Dirac operator, and corresponds to $(9.10)$ in $[6]$.

\section{Acknowledgments}

We thank Martin Möller, Frankfurt, for providing a proof of Lemma 3.1 using Teichmüller theory. His proof was an inspiration for the argument in the case $n=2$ presented above. We also thank the referee for many helpful suggestions.

\section{References}

[1] B. Ammann, M. Dahl and E. Humbert, Surgery and harmonic spinors, Adv. Math. 220(2) (2009), 523-539.

[2] N. Anghel, Generic vanishing for harmonic spinors of twisted Dirac operators, Proc. Amer. Math. Soc. 124(11) (1996), 3555-3561.

[3] C. Bär and M. Dahl, Surgery and the spectrum of the Dirac operator, J. Reine Angew. Math. 552 (2002), 53-76.

[4] A. L. Besse, Einstein manifolds, in 'Ergebnisse der Mathematik und ihrer Grenzgebiete (3)', 10, Springer-Verlag, Berlin, 1987.

[5] B. Booß-Bavnbek and M. Lesch, The invertible double of elliptic operators, Lett. Math. Phys. 87(1-2) (2009), 19-46.

[6] B. Booß-Bavnbek and K. P. Wojciechowski, Elliptic boundary problems for Dirac operators, in 'Mathematics: Theory \& Applications', Birkhäuser Boston Inc., Boston, MA, 1993.

[7] C.J. Earle and J. Eells, The diffeomorphism group of a compact Riemann surface, Bull. Amer. Math. Soc. 73 (1967), 557-559. 
[8] T. Friedrich, Dirac operators in Riemannian geometry, in 'Graduate Studies in Mathematics', 25, AMS, Providence, RI, 2000.

[9] R. C. Kirby, The topology of 4-manifolds, in 'Lecture Notes in Mathematics', 1374, SpringerVerlag, Berlin, 1989.

[10] A. A. Kosinski, Differential manifolds, in 'Pure and Applied Mathematics', 138, Academic Press Inc., Boston, MA, 1993.

[11] H. B. Lawson, Jr. and M.-L. Michelsohn, Spin geometry, in 'Princeton Mathematical Series', 38, Princeton University Press, Princeton, NJ, 1989.

[12] S. Maier, Generic metrics and connections on Spin- and Spin ${ }^{c}$-manifolds, Comm. Math. Phys. 188(2) (1997), 407-437.

[13] S. Stolz, Simply connected manifolds of positive scalar curvature, Ann. Math. (2) 136(3) (1992), $511-540$.

[14] A. J. Tromba, Teichmüller theory in Riemannian geometry, in 'Lectures in Mathematics ETH Zürich', Birkhäuser Verlag, Basel, Lecture notes prepared by Jochen Denzler, 1992.

Fakultät für Mathematik, Universität Regensburg, 93040 Regensburg, Germany

E-mail address: bernd.ammann@mathematik.uni-regensburg.de

$U R L:$ http://www. berndammann. de

Institutionen för Matematik, Kungliga Tekniska Högskolan, 10044 Stockholm, Sweden

E-mail address: dahl@math.kth.se

Laboratoire de Mathématiques et Physique Théorique Université de Tours UFR Sciences et Techniques Parc de Grandmont, 37200 Tour - FRANCE

E-mail address: humbert@lmpt.univ-tours.fr 